Conclusion* Ovarian cancer in premenopausal women is a threatening condition, diagnosed in most cases in advanced stages, with a high probability of bad prognosis despite appropriate surgical and oncologic management.

\section{PROGNOSTIC IMPACT OF PD-L1 EXPRESSION IN EPITHELIAL OVARIAN CANCER: A COHORT OF 49 PATIENTS}

${ }^{1} \mathrm{D}$ Atallah*, ${ }^{2} \mathrm{~A}$ Khaddage, ${ }^{2} \mathrm{~L}$ Khoury, ${ }^{1} \mathrm{~W}$ Abdallah, ${ }^{2} \mathrm{~V}$ Smayra, ${ }^{3} \mathrm{M}$ Akiki, ${ }^{1} \mathrm{~N}$ El Kassis, ${ }^{4} \mathrm{G}$ Chahine, ${ }^{1} \mathrm{M}$ Moubarak. ${ }^{1}$ Hôtel-Dieu de France - Saint Joseph University, Obstetrics and Gynecology, Beirut, Lebanon; ${ }^{2}$ Hôtel-Dieu de France - Saint Joseph University, Pathology, Beirut, Lebanon; ${ }^{3}$ Hôtel-Dieu de France, Pathology, Beirut, Lebanon; ${ }^{4}$ Hôtel-Dieu de France - Saint Joseph University, Oncology, Beirut, Lebanon

\subsection{6/ijgc-2021-ESGO.391}

Introduction/Background* Role of checkpoint inhibitors in ovarian cancer is still unknown and results from ongoing clinical trials are still awaited. We aim in this study to assess the expression of PD-L1 using the Combined Positive Score (CPS) and to evaluate its impact on the overall survival in a cohort of 49 patients diagnosed with high-grade serous ovarian cancer.

Methodology Medical charts were reviewed of 49 patients with high-grade serous ovarian cancer operated on at the gynecologic oncology department in Hôtel-Dieu de France hospital, Lebanon, between 2015 and January 2020. Immunohistochemical staining was performed for PD-L1 (Agilent Dako, PDL-1 IHC 22C3) and for TP53 (Agilent Biogenex, clone D07, 1:100 dilution) on whole tissue sections from a representative block of formalin-fixed, paraffin-embedded tumor tissue. We looked for correlation between PD-L1 status and overall survival/recurrence. We also looked for correlation between PD-L1 status and TP-53 mutation.

Result(s)* 55\% of patients presented a positive PD-L1 status. No correlation was found between the PD-L1 status and the stage of the disease. Lymph node status was similar between the two cohorts, positive vs. negative CPS score $(p=0.927)$. Median follow-up was 36 months (range, 12 - 72 months). Survival rate was similar between the two cohorts, positive vs. negative PD-L1 status $(88.9 \%$ vs. $72.7 \%$ respectively, $\mathrm{p}=$ $0.14)$. No correlation was found between recurrence rate and PD-L1 status ( $p=0.184)$. Also, no correlation was found between PD-L1 status and TP-53 type (wild vs. mutated) $(\mathrm{p}=$ $0.154)$

Conclusion* PD-L1 status does not seem to have a prognostic impact in our series of patients with high-grade serous epithelial ovarian cancer. Also, patients with TP53 mutation do not present increased expression of PD-L1 in comparison to patients with TP53 wild-type.

\section{CLINICAL BENEFIT OF ORAL METRONOMIC CYCLOPHOSPHAMIDE ADMINISTRATION IN HEAVILY PRETREATED RECURRENT EPITHELIAL OVARIAN CANCER}

${ }^{1} \mathrm{D}$ Attianese*, ${ }^{1} \mathrm{E}$ Badellino, ${ }^{1} \mathrm{M}$ Villa, ${ }^{2} \mathrm{M}$ Bellero, ${ }^{1} \mathrm{R}$ Massobrio, ${ }^{1} \mathrm{~L}$ Fuso, ${ }^{1} \mathrm{~N}$ Biglia, ${ }^{1}$ A Ferrero. 'Mauriziano Hospital, Academic Department Gynaecology and Obstetrics, Torino, Italy; ${ }^{2}$ Mauriziano Hospital, Hospital Pharmacy, Torino, Italy
Introduction/Background* Oral metronomic cyclophosphamide (OMC) consists in the chronic administration of low, usually daily, doses of chemotherapy. The effective reduction of tumor growth, oral administration, low toxicity profile and low cost make this therapeutic choice attractive for women with relapsed ovarian cancer, especially heavily pretreated patients. We retrospectively evaluated the clinical benefit and the objective response in patients treated with OMC for recurrent ovarian cancer.

Methodology We included patients treated with OMC $(50 \mathrm{mg}$ daily) from 2016 to 2021 at the Academic Department Gynaecology, Mauriziano Hospital, Torino, Italy. Clinical benefit was classified as CA125 response, radiological response and symptomatic improvement as reported by the treating physician. Toxicity profile was assessed using Common Terminology Criteria for Adverse Events version 5.0.

Result(s)* Thirty-eight patients were analyzed. Average age was 72 years (range 49-88). 34 (90\%) had FIGO stage III and IV disease at diagnosis and $64 \%$ had received $\geq 3$ previous lines. Before starting OMC, 21\% had ECOG 0, 58\% ECOG 1 and 21\% ECOG 2. $8.6 \%$ of patients obtained partial response (PR) and 37\% stable disease (SD). Median duration of the response was 7.4 months. After 3 months from starting OMC, $51 \%$ of patients experienced symptoms improvement and $69.5 \%$ Ca125 reduction or stabilization. Only one patient discontinued for side effects and no G3-4 hematological toxicities were observed, reflecting a low toxicity profile. Only nausea and fatigue G1-G2 were reported in $5(13 \%)$ and 13 (34\%) cases, respectively.

Conclusion* OMC could be a feasible therapy for recurrent ovarian cancer leading to an acceptable clinical response with a low toxicity profile, even if patients are heavily pretreated and with a suboptimal performance status. We are now prospectively assessing the Patient Reported Outcomes (PROs) in order to objectify the tolerability and the symptoms improvement.

All authors have no conflict of interest.

\section{PLATELET COUNT AS A PROGNOSTIC INDICATOR IN OVARIAN CANCER}

H Dubey*, A Ranjan, P Tanwar. Dr.B.R.A.IRCH, AllMS, New Delhi, Laboratory Oncology, New Delhi, India

\subsection{6/ijgc-2021-ESG0.393}

Introduction/Background* Thrombocytosis is a poor prognostic indicator in malignancies especially in gynecological one such as carcinoma ovary, cervix, and endometrium. Recent evidence indicates that platelets are present in the tumor microenvironment and could play important roles in stimulating tumor growth. The aim of this study is to analyze the impact of platelet count on the prognosis of ovarian cancer

Methodology Baseline platelet count (prior to surgery or chemotherapy) was analyzed in 151 cases of ovarian cancer confirmed by histopathological examination. Thrombocytopenia \& thrombocytosis were defined as platelet count (PC) $<1.0$ lakh $\&>4.0 \mathrm{lakh} / \mathrm{cumm}$ respectively.

Result(s)* Out of 151 cases, thrombocytopenia was seen in $3.3 \%(5 / 151)$, whereas thrombocytosis in 20.5\% (31/151) and $76.2 \%(115 / 151)$ of cases were within normal range of platelet count. 